\title{
Optimal Energy Measurement in Nonlinear Systems: An Application of Differential Geometry
}

\author{
D. J. Fixsen • S. H. Moseley • \\ T. Gerrits · A. E. Lita · S. W. Nam
}

Received: 22 August 2013 / Accepted: 22 February 2014 / Published online: 21 March 2014 (C) Springer Science+Business Media New York (outside the USA) 2014

\begin{abstract}
Design of TES microcalorimeters requires a tradeoff between resolution and dynamic range. Often, experimenters will require linearity for the highest energy signals, which requires additional heat capacity be added to the detector. This results in a reduction of low energy resolution in the detector. We derive and demonstrate an algorithm that allows operation far into the nonlinear regime with little loss in spectral resolution. We use a least squares optimal filter that varies with photon energy to accommodate the nonlinearity of the detector and the non-stationarity of the noise. The fitting process we use can be seen as an application of differential geometry. This recognition provides a set of well-developed tools to extend our work to more complex situations. The proper calibration of a nonlinear microcalorimeter requires a source with densely spaced narrow lines. A pulsed laser multi-photon source is used here, and is seen to be a powerful tool for allowing us to develop practical systems with significant detector nonlinearity. The combination of our analysis techniques and the multi-photon laser source create a powerful tool for increasing the performance of future TES microcalorimeters.
\end{abstract}

Keywords TES $\cdot$ X-ray $\cdot$ Microcalorimeter - Optimal filter $\cdot$ Nonlinear response · Non-stationary noise $\cdot$ Differential geometry

Contribution of NIST, an agency of the U.S. Government, not subject to copyright.

\author{
D. J. Fixsen · S. H. Moseley ( $\varangle)$ \\ Goddard Space Flight Center, Greenbelt, MD, USA \\ e-mail: harvey.moseley@nasa.gov \\ D. J. Fixsen \\ e-mail: dale.j.fixsen@nasa.gov \\ D. J. Fixsen \\ University of Maryland, College Park, MD, USA \\ T. Gerrits · A. Lita $\cdot$ S. W. Nam \\ National Institute of Standards and Technology, Boulder, CO, USA
}




\section{Introduction}

Microcalorimeters have become the detectors of choice for high efficiency, high resolution energy spectroscopy for applications ranging from X-ray spectroscopy to nuclear nonproliferation studies. In the last twenty years, most experimenters have moved from semiconductor-based thermometers to the more sensitive thermometers made using the superconductor-normal transition. The more sensitive thermometer offers an improvement in energy resolution at the price of a limited linear dynamic range. Typically, experimenters have chosen to add heat capacity to the detectors to assure the highest energy events fall in the linear regime, reducing the resolving power of the detector. We present a set of analysis techniques that demonstrate that the TES detectors can be operated far into the nonlinear regime where the noise is significantly non-stationary [1], and still produce energy resolution close to that seen in the linear regime. These tools remove design constraints, allowing a new generation of higher performance detectors.

The analysis process requires calibration events or a detailed physical model of the detector over a range of energies, ranging from the linear regime to the maximum required energy. For this work, our source is an attenuated laser pulse with a welldefined average photon number $\langle n>[2,3]$. Due to the Poisson distribution of photon number in the weak laser pulse, a given laser pulse strength will produce a comb of varying photon number around the mean photon number $\mathrm{n}$. By varying the mean photon number in a pulse, we can cover the full required energy range with a comb of events of accurately known energy. Given these events we calculate several objects that characterize the signal and noise of the system: (1) the average pulse shape at a given energy. (2) the residual pulse at each energy after removal of the average pulse. (3) the covariance matrix of the residual time series at each energy and the weight matrix, which is the inverse of the covariance. Given these objects, we can create least squares optimal filters for measuring the energy difference between events over the full energy range, the primary objective of spectroscopy. We present the technique and apply it to a data set of laser pulse events.

\section{Overview}

Here we present analysis of pulses on a TES bolometer. A fiber-coupled, pulsed laser diode was used to illuminate a tungsten TES detector. The average photon number per pulse was controlled using programmable fiber attenuators. A schematic of the experiment is shown in Fig. 1 [3].

For our study we used a TES consisting of a $25 \mu \mathrm{m} \times 25 \mu \mathrm{m} \times 20 \mathrm{~nm}$ tungsten film with a superconducting transition temperature of about $130 \mathrm{mK}$. We mounted the TES inside a commercial dilution refrigerator (DR) at the mixing chamber stage at about $20 \mathrm{mK}$. Using a single-mode $630 \mathrm{~nm}$ fiber $(4.0 \mu \mathrm{m}$ mode field diameter) we coupled the light pulses from the attenuated laser at room temperature to the detector. At the mixing chamber, we coiled the fiber around a $1 / 4$ brass rod to eliminate any long-wavelength background photons, i.e. black body photons [4]. The single-mode 


\section{$405 \mathrm{~nm}$ laser}

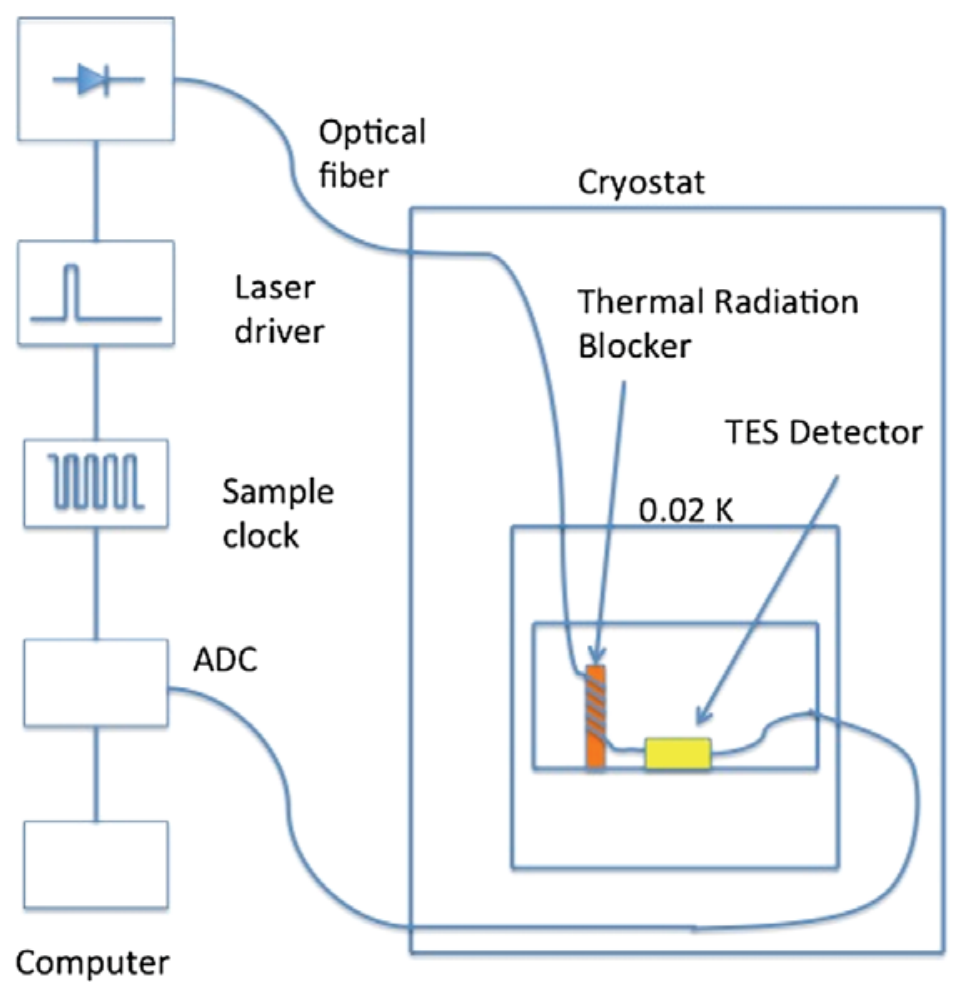

Fig. 1 This shows a schematic of the measurement apparatus. Not shown is the programmable attenuator used to control the mean photon number per pulse absorbed in the TES detector. The optical fiber was coiled tightly near the detector to filter out the blackbody radiation that is coupled into the fiber at the top of the cryostat The response of the TES to each pulse is sampled at $50 \mathrm{MHz}$ and recorded in a file. This process is repeated approximately 10,000 times per second (Color figure online)

fiber was self-aligned to the detector [5]. The light pulses were delivered from a 405 $\mathrm{nm}$ commercial laser pulsed with an external function generator.

The signals from the TES detectors were digitized at $50 \mathrm{Msamples} / \mathrm{sec}$. For the analysis presented, we used 50 samples before the photon pulses arrived and 350 samples of the signal pulse. Obviously the data could be Fourier transformed to form a data set which is a function of frequency instead of samples as a function of time. This is just a change of basis, the same vectors can be expressed equally well in this basis. Indeed there are an infinite number of basis sets that could be used to express the same vectors. We refer to the vector without being overly concerned about the basis in which the vector is expressed. A vector is just a point in this 400 dimensional vector space. A typical pulse is shown in Fig. 2. Approximately $10^{6}$ such pulses were collected.

For convenience, we use Einstein summation notation in this paper. The same variable names are used for the contravariant and covariant cases even though the numerical values are different, because the underlying information is the same. Hence a sample of data, such as in Fig. 2, is denoted $D^{i}$.

\section{Linear Approximation}

An average pulse, $A^{i}=\left\langle D^{i}>\right.$, (see Fig. 3) can be used as a template to form a matched filter. If this average pulse is multiplied by the data in a vector sense (dot 


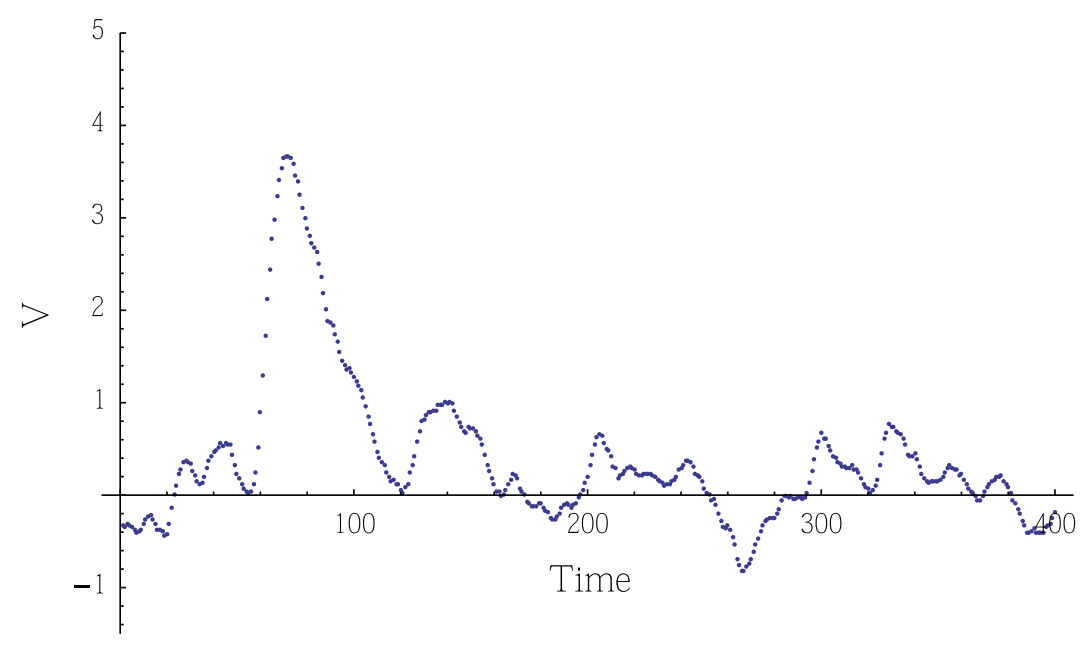

Fig. 2 Typical response of the TES to a a single $405 \mathrm{~nm}$ photon. The vertical scale is arbitrary, and the $\mathrm{X}$-axis is labeled by the number of $20 \mathrm{~ns}$ samples (Color figure online)

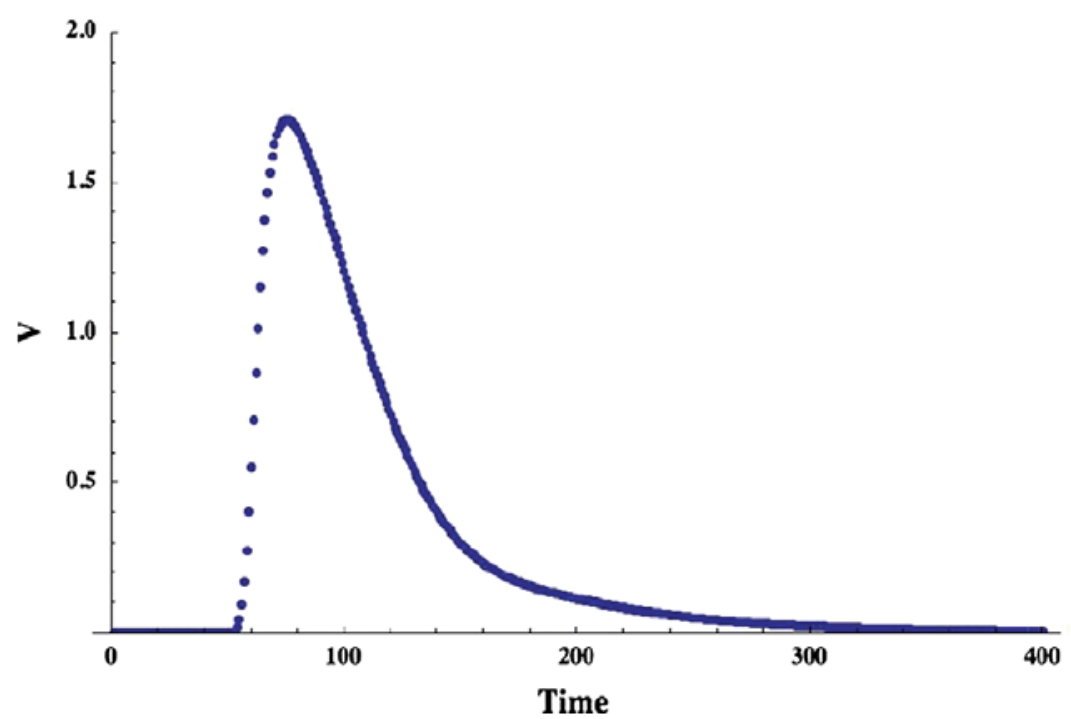

Fig. 3 Average of all responses to an average rate of $\sim 3$ photons per pulse. The vertical scale is arbitrary, and the $\mathrm{X}$-axis is labeled by the number of $20 \mathrm{~ns}$ samples (Color figure online)

product) $r=A \cdot D$, the resulting histogram clearly shows distinct peaks at different numbers of photons (see Fig. 4). But this implicitly assumes that the samples are uncorrelated.

Noise records are taken and the resulting data is used to find a covariance matrix $V^{i j}=<D^{i} D^{j}>$. With a sufficient number of data samples, this matrix, contains a complete description of the noise characteristics of the detector in the linear regime (see Fig. 5). The covariance matrix then represents an error ellipsoid around the vector that is the limit of our knowledge of the "true" position of a vector. The least squares optimal weight matrix is the inverse of the covariance matrix $W_{i j}=\left(V^{i j}\right)^{-1}$.

The pulses with a single photon can be selected from the entire set shown in Fig. 4 and averaged to form a template for a single photon pulse, $S$. A better fit can be formed by $r=S^{i} W_{i j} D^{j} /\left(S^{k} W_{k l} S^{l}\right)$. The denominator is included to normalize the result to the number of photons (see Fig. 6). 


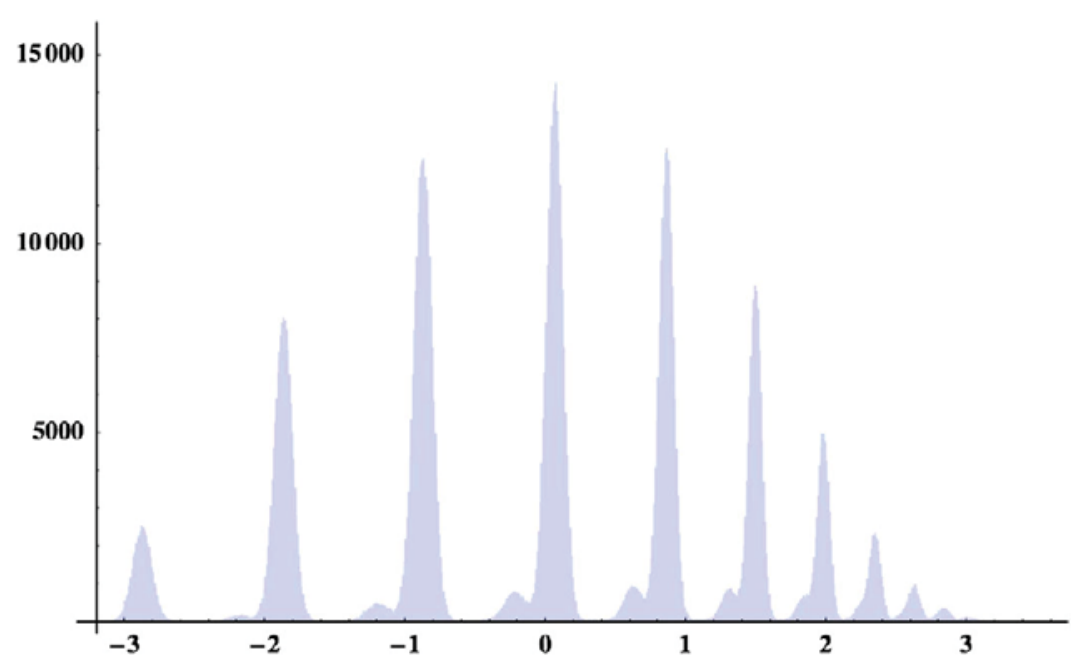

Fig. 4 Distribution of the dot product of the mean-subtracted average pulse with each pulse. The different peaks are from events with different photons number, and their number is Poisson distributed as expected (Color figure online)

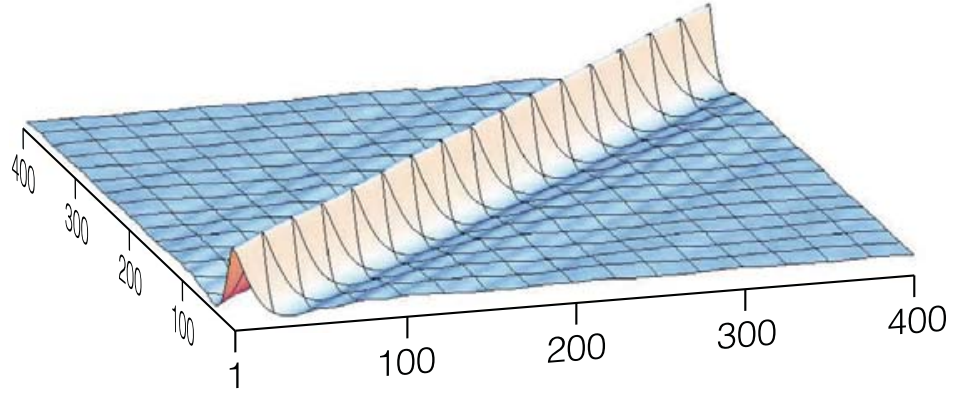

Fig. 5 Covariance of our 400-sample noise records $\left.\left(<x^{i} x^{j}\right\rangle\right)$ in the absence of pulses. As expected the noise is stationary and the value of the covariance matrix is set by the distance to the main diagonal. The finite width shows that we substantially oversample the detector (Color figure online)

A filter, $F_{j}=S^{i} W_{i j} /\left(S^{k} W_{k l} S^{l}\right)$, can be preformed so the fit can be made with a single inner product for each pulse. Note the peaks for 1,2 etc photons are distinct and clearly separated. Indeed since the frequency (hence energy) of a single photon is known, the filter can be calibrated by multiplying the pulse by the photon energy, $h v$ or $E_{j}=h v F_{j}$. Since this paper is concerned with the analysis process, the energy is calibrated in $405 \mathrm{~nm}$ photons.

It can be seen in Figs. 4, 6, and 10 that there is a shoulder to the left of each peak. This may arise from fluorescent loss in the fiber or from a loss mechanism in the detector. This is consistent with a model where each photon has a probability of $\sim 2 \%$ of losing $\sim 1 \mathrm{eV}$ of energy, resulting in the small shoulder that is observed. There is no significant difference in the shapes of the main peak and shoulder pulses.

\section{Non-linear Region}

As the mean number of photons per pulse is increased the optimal detection filter for a single photon pulse no longer works as well. There are two reasons for this. The first is that the shape has changed and $S^{i}$ is no longer the correct shape. Figure 7 shows the average pulse for one photon ${ }^{1} P^{i}=S^{i}$, two photons ${ }^{2} P^{i}$ and so on. 


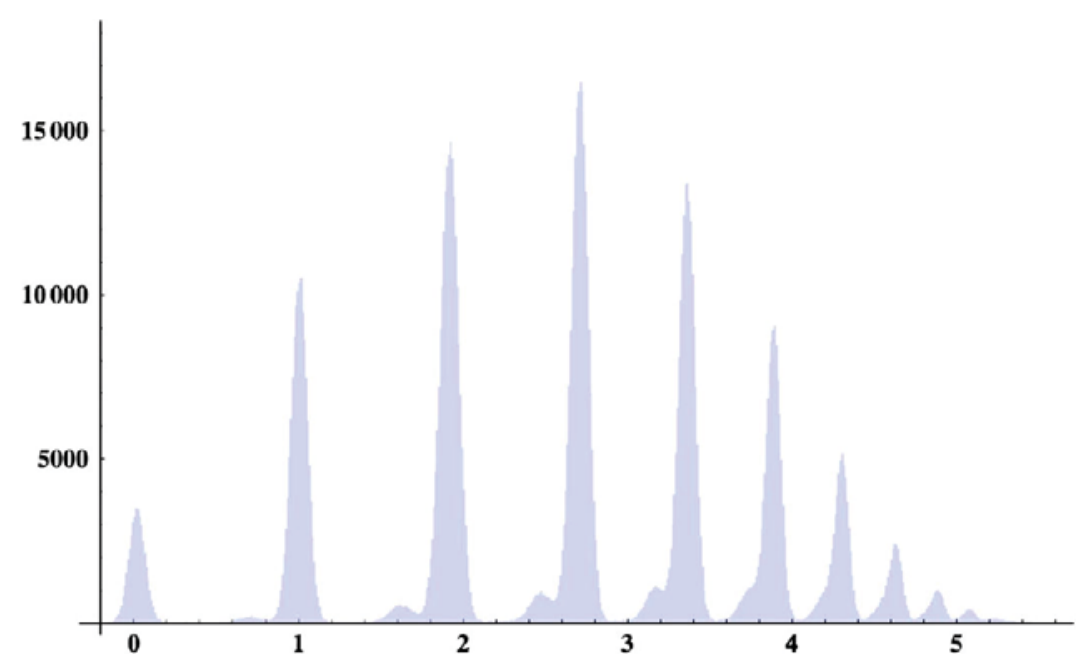

Fig. 6 Pulse height distribution using the one photon average signal and weight matrix from the noise records to generate the linear optimal filter. The peak of the one photon pulses is at 1 as expected, but higher peaks are not uniformly spaced due to the non-linear response of the TES (Color figure online)

A second problem is that the noise characteristics have changed and the linear weight matrix $W_{i j}$ is no longer optimal (see Fig. 8).

Both of these things can be fixed. What is needed is a differential photon response, $\delta^{i}=d P^{i} / d E$ that properly describes the direction of energy change in this space. We can use ${ }^{n} \Delta^{i}={ }^{n} P^{i}-{ }^{n-1} P^{i}$, shown in Fig. 9, as an approximation for the true differential response.

Let us call the weight matrix we have derived ${ }^{0} W_{i j}$. Now another weight matrix ${ }^{1} W_{i j}$ can be derived from the dispersion of the pulses with one photon. Next, a second energy filter ${ }^{2} E_{j}=\frac{{ }^{2} \Delta^{i}{ }^{1} W_{i j}}{{ }^{2} \Delta^{k}{ }^{1} W_{k l}{ }^{2} \Delta^{l}}$ is defined. The divisor is included to normalize the filter, so 1 means one additional photon.

At this point is worth considering what we have done in mathematical terms. In the initial case we took the covariance matrix to be the dispersion with no photons present. This is equivalent to constructing a tangent plane at zero energy, and using the tangent plane so constructed to project the distance along the pulse shape direction.

Next we constructed a tangent plane at the point where the input energy is a single photon. The direction (and distance) from that point to where the signal equals two photons is mathematically ${ }^{2} \Delta^{j}={ }^{2} P^{j}-{ }^{1} P^{j}$.

Obviously one can continue this process for each additional photon. As long as we can resolve resolve $n$ from $n+1$ photons, the process can continue, although interloper events from another energy can badly affect the covariance matrix. We used records from the upper half of the main peaks to assure that all events arose from the same energy deposition. The result of this process from zero to to $n=6$ is shown in Fig. 10 . The number of 6 photon events is much smaller than the number of 3 photon events, but this could be easily remedied by changing the attenuator to increase the mean photon number.

In general the covariance can be expressed as a function of energy $V^{i j}(E)$. This function expresses part of the curvature of the data space. Since the non-linearity of the 


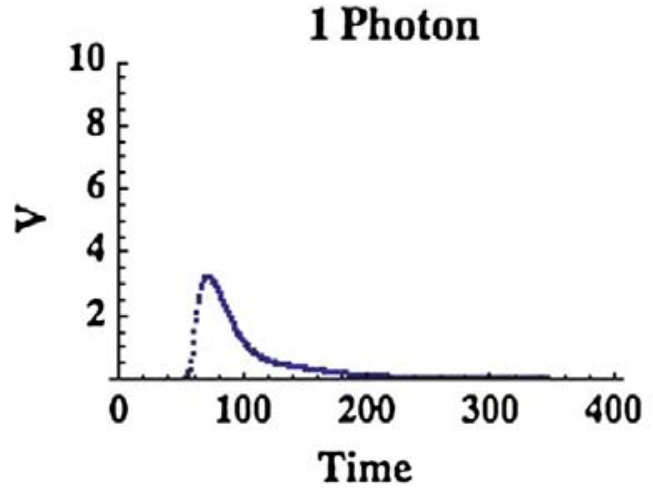

3Photons

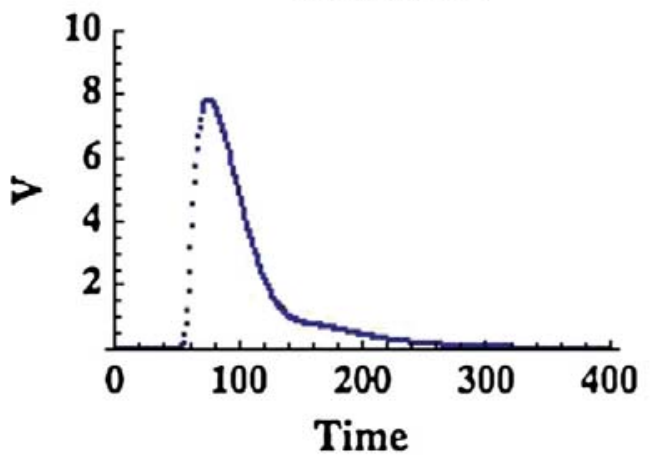

5 Photons

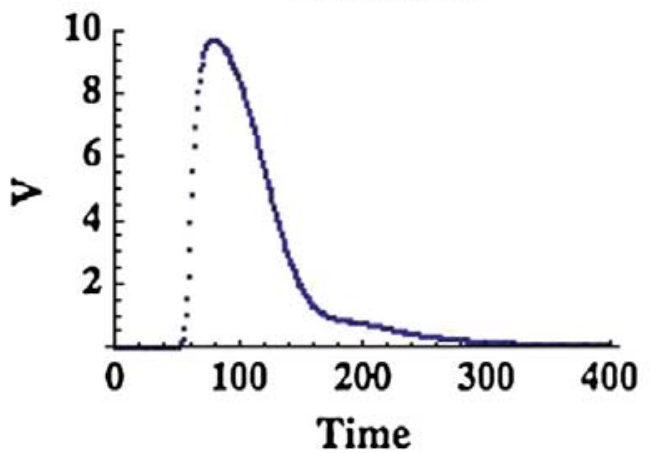

2 Photons

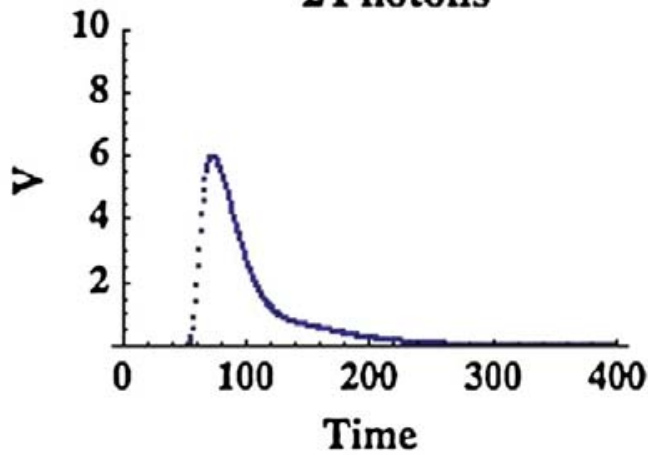

4Photons

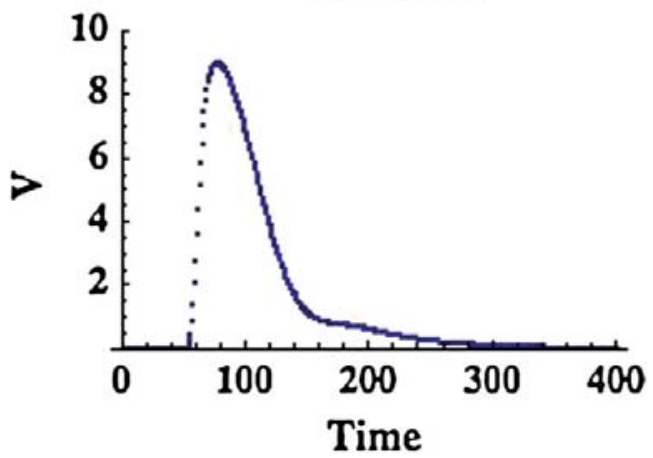

6Photons

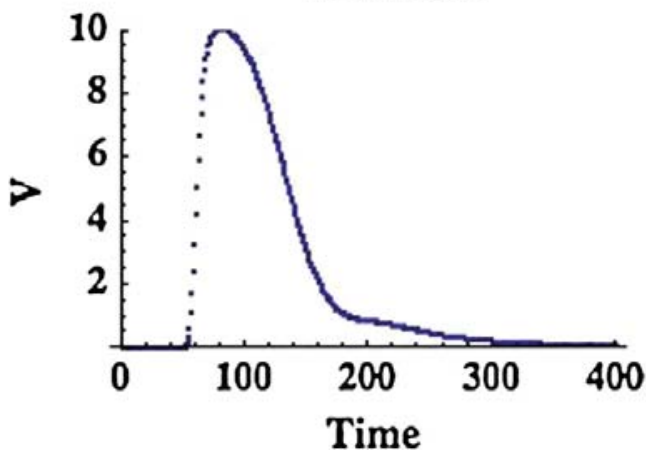

Fig. 7 Pulse shapes for n-Photon pulses ranging from 1 to 6 . The vertical scale is arbitrary but is the same as in Fig. 9. By 4 photons the TES is saturated. Thereafter the pulse grows in duration but not in height as the temperature of the TES near the normal transition (Color figure online)

system is primarily driven by the temperature rise to beyond the critical temperature, most of the curvature of the covariance matrix will be along this direction.

Other measurements can be made to fill in between the points explored by the photons in this experiment, or the covariance matrices and pulse shapes can be interpolated to the regions between an integral number of photons.

Figure 11 shows that the energy resolution degrades with higher energy but only modestly even though by 6 photons the TES is effectively driven normal and the pulse shape is very different from the single photon pulse shape. If one uses the linear optimal filter in the nonlinear regime, there is about a factor of two loss in resolution for the 6-photon events. 

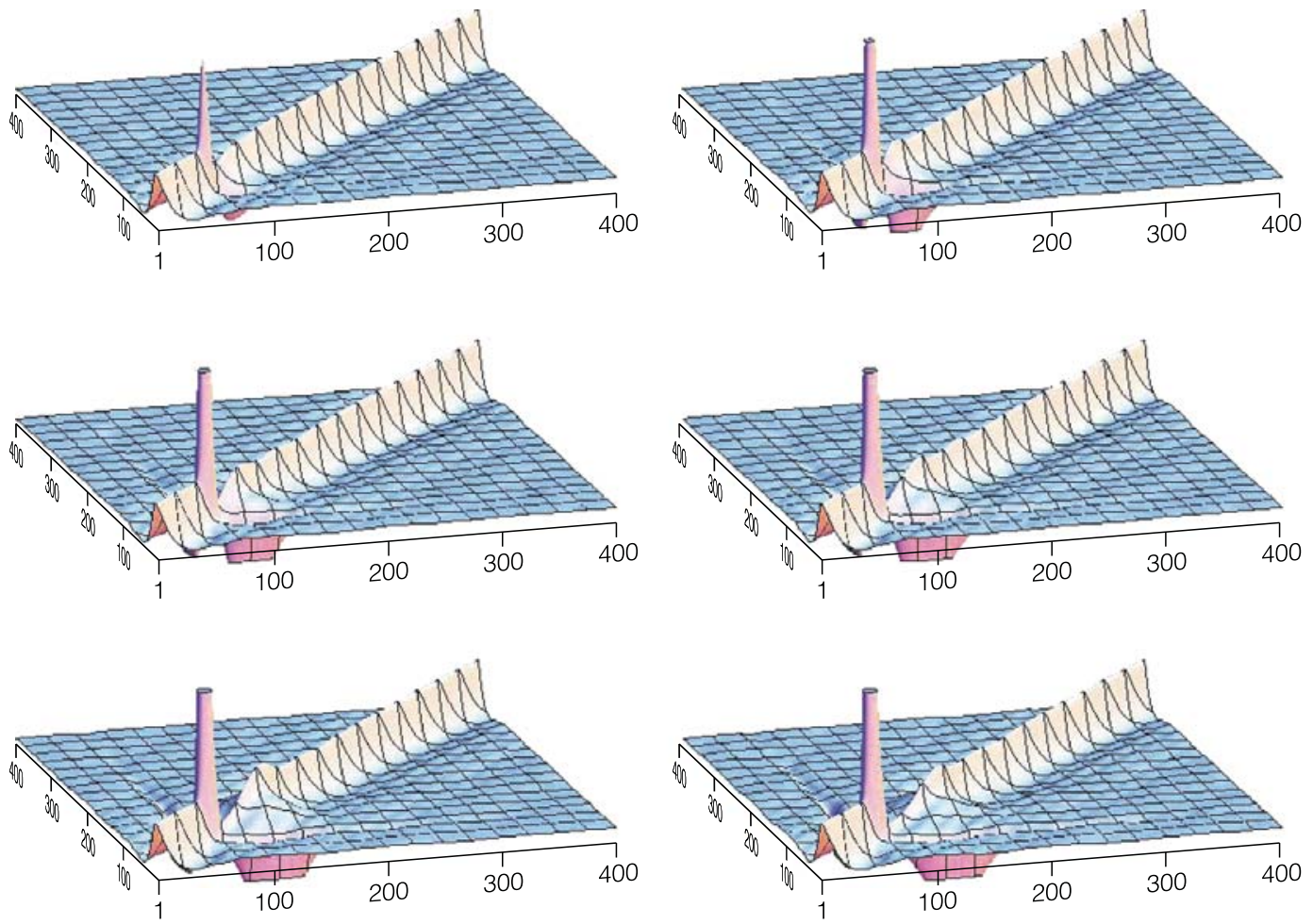

Fig. 8 Covariance $\left(<x^{i} x^{j}>\right.$ ) of residual noise for events ranging from 1 to 6 photons. The covariance shows a peak (clipped in this display) at the rise of the pulse. This may indicate a variation of arrival time. For the larger pulses there is a lower noise after the initial rise while the TES is saturated. This shows the noise is no longer stationary (Color figure online)

\section{Other Parameters}

There is obviously a change in covariance along the energy dimension (energy). Other dimensions might also exhibit a variable covariance (e.g. phase or time of the pulse from a fiducial sample, or position of the photon absorption on the microcalorimeter). As these other dimensions are explored a full function of the covariance matrix could be constructed.

The general case can have many parameters. For example, a delay or phase could be introduced in addition to the energy. To measure this, an electronic delay in the pulse or in the digitization could be introduced. Alternatively a phase could be modeled as a shift in the pulse, $\theta^{i}=P^{i+1}-P^{i}$, with appropriate care at the end points. An optimal filter for the phase shift is $\theta_{i}=W_{i j}\left(P^{j+1}-P^{j}\right) /\left(\left(P^{k+1}-P^{k}\right) W_{k l}\left(P^{l+1}-P^{l}\right)\right)$ where the divisor is included so that the result is 1 when the phase shift is equal to one time period.

In general many parameters can be fit and we will index these parameters with $\mu, v$, etc. Then we can see that for a particular parameter, $X, H_{\mu}^{i}=\partial D^{i} / \partial X$. Then a complete description of the pulse in terms of the parameters is $P^{\mu}=$ $\left(H_{\mu}^{i} W_{i j} H_{v}^{j}\right)^{-1} H_{v}^{k} W_{k l} D^{l}$ or $P_{\mu}=H_{\mu}^{i} D_{i}$ in short hand notation.

Here we have shown that $W$ is a function of the energy. The well known mathematics of differential geometry $[6,7]$ allows us to calculate the appropriate terms. The weight matrix that describes the noise is the metric tensor for the space, and changes of the weight matrix with signal amplitude results in curvature of that space. The changes 

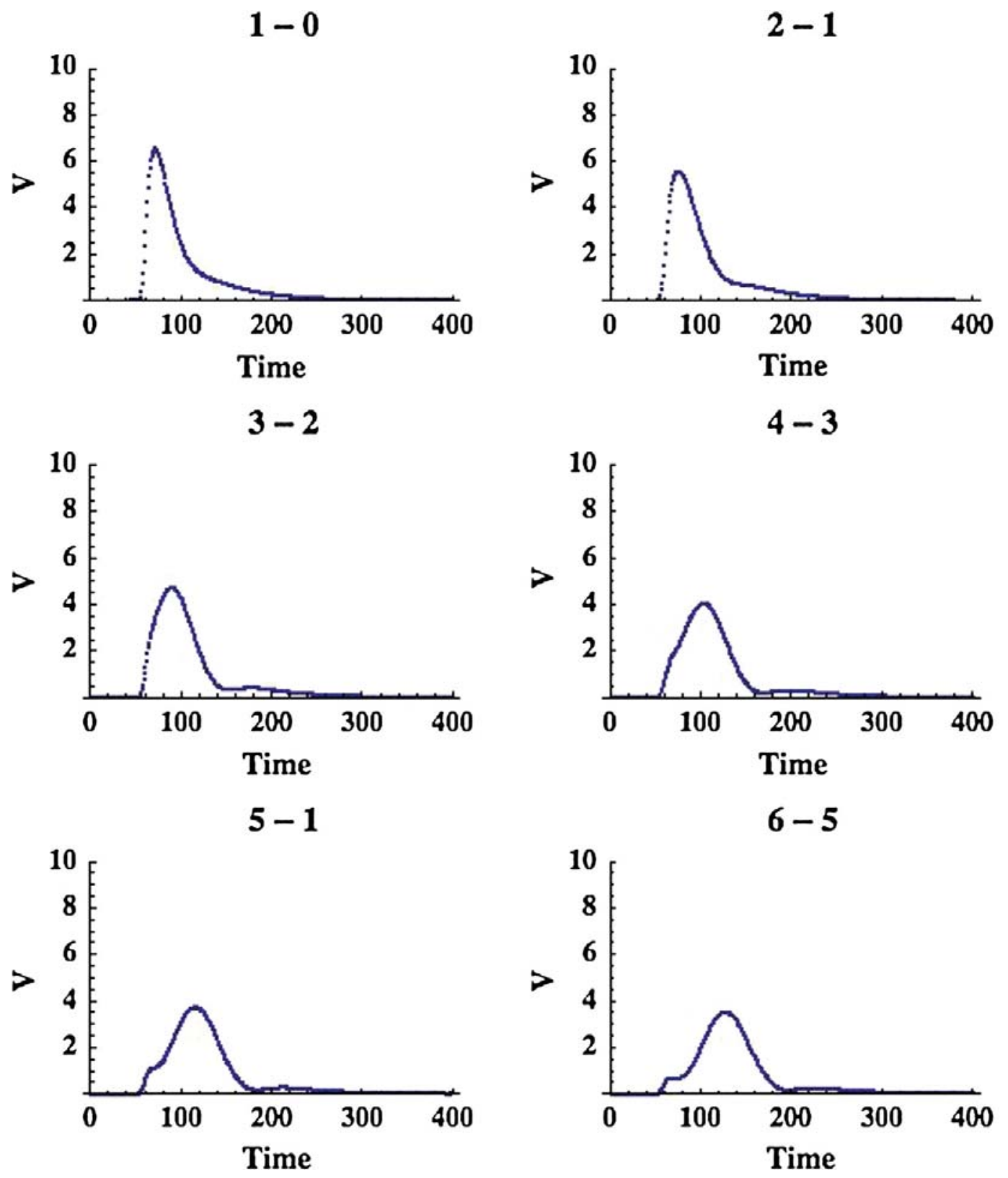

Fig. 9 Differential pulse shapes for pulses of $n$ and $n-1$ photons. The vertical scale is arbitrary but is the same as in Fig. 7. This emphasizes the variation in pulse shape as the pulse grows in duration but not as much in height (Color figure online)

in the direction of the signal vectors with signal amplitude shows the curvature of the model space. One can use standard procedures to calculate the curvature of the space to allow accurate interpolation of the model and data spaces, providing optimal filtering at all energies.

Finally, in defining the data vector, there is no requirement that the data be of a similar kind. We can add other dimensions to our data vector which might be the detector temperature measurement, bias voltage, or some other parameter which might be of interest. The process becomes a powerful framework for organizing our knowledge of our entire experiment and in removing systematic effects from our parameter determination. 


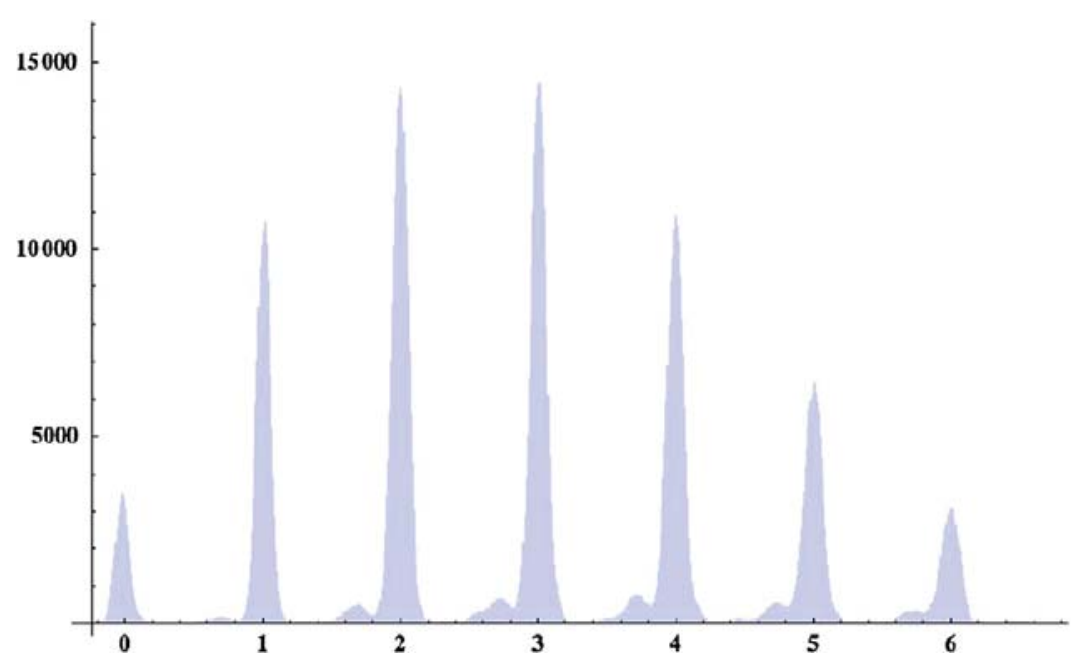

Fig. 10 Energy response to 1-6 photons calibrated at each photon number (Color figure online)

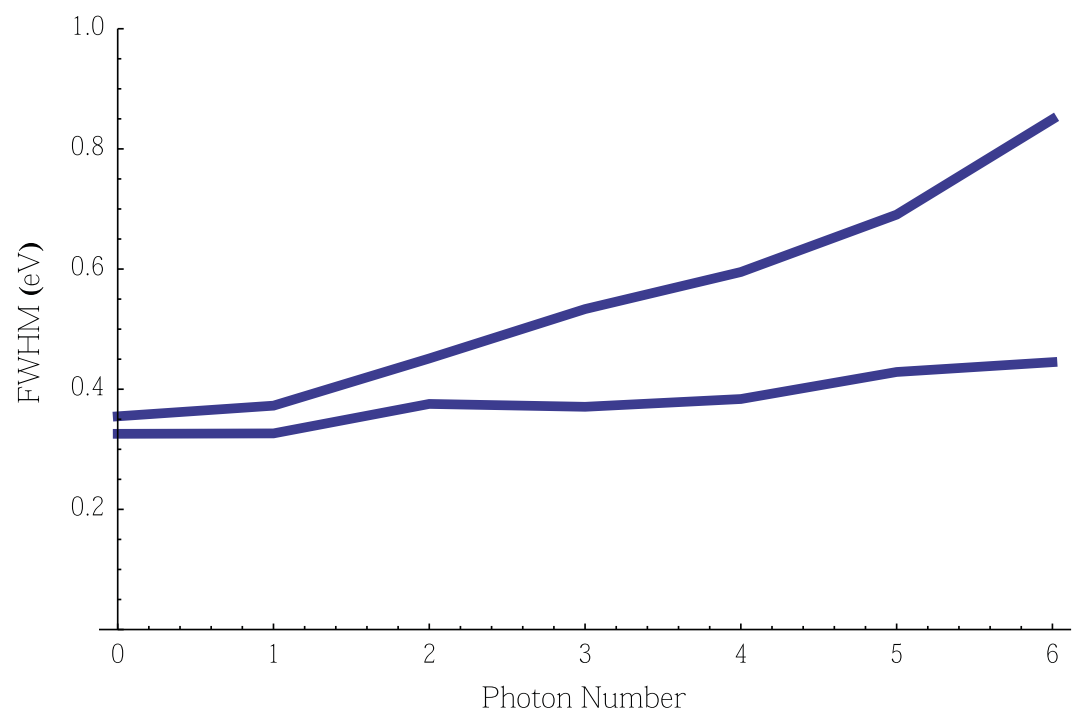

Fig. 11 Energy resolution as a function of the number of photons per pulse. The upper line shows the resolution achieved using a linear optimal filter using the one-photon pulse shape and pulse-free noise records. The decrease in energy resolution with increasing energy is caused by both the failure to use the correct differential pulse shape at each energy, and the failure to include changes in noise with increasing energy. This noise increase can be either due to the changes in detector parameters in the nonlinear regime, or due to unmodeled variance, such as rise time jitter. The lower line displays the resolution achieved using the formalism introduced in this paper. The increase in FWHM with photon number represents an upper limit to the actual change, since we know we have a significant unmodeled source of variance in the detector, the arrival time of the pulse (Color figure online)

\section{Sensitivity to Parametric Changes}

There is a concern that the extraction of spectra in the nonlinear regime will be more sensitive to operational parameters than in the conventional linear case. The parameters of greatest concern are the base temperature of the detector and the applied bias. In the linear case, either of these variations can produce first order changes in both the pulse height and the time constant of the detector. Typically, one analyzes the events 
with a filter designed for the unperturbed conditions and corrects the spectrum by normalization. It is expected that for small changes in parameter that the linear correction described will be adequate to rectify the perturbed spectrum, but a calibration source with a range of lines such as used in this work will be required. With lines covering the full spectral range, the linear correction in pulse energy can be derived and applied, much as in the case of the linear system. Our approach is based on local linearization, and should not depend sensitively on small perturbations.

Acknowledgments We would like to thank Sarah Busch, Simon Bandler, and Steve Smith for helpful discussions about nonlinear detector response, and Dan McCammon for a wide range of helpful discussions.

\section{References}

1. D.J. Fixsen, S.H. Moseley, B. Cabrera, E. Figueroa-Feliciano, Pulse estimation in nonlinear detectors with nonstationary noise. Nucl. Instr Methods Phys. Res. A 520, 555-558 (2004)

2. A.E. Lita, A.J. Miller, S.W. Nam, Counting near-infrared single-photons with $95 \%$ efficiency. Opt. Express 16, 3032 (2008)

3. T. Gerrits, B. Calkins, N. Tomlin, A.E. Lita, A. Migdall, R. Mirin, S.W. Nam, Extending single-photon optimized superconducting transition edge sensors beyond the single-photon counting regime. Opt. Express 20, 23798 (2012)

4. D. Rosenberg, A.E. Lita, A.J. Miller, S.W. Nam, Noise-free high-efficiency photon-number-resolving detectors. Phys. Rev. A 71, 061803 (2005)

5. A.J. Miller, A.E. Lita, B. Calkins, I. Vayshenker, S.M. Gruber, S.W. Nam, Compact cryogenic selfaligning fiber-to-detector coupling with losses below one percent. Opt. Express 19, 9102-9110 (2011)

6. P.A.M. Dirac, General Theory of Relativity (Princeton University Press, Princeton, 1996)

7. C. Misner, K. Thorne, J. Wheeler, in Gravitation, vol. 3, ed. by W. H. Freeman (1973) 